Kragujevac Journal of Mathematics

Volume 38(1) (2014), Pages 203-222.

\title{
ODD SUM LABELING OF SOME SUBDIVISION GRAPHS
}

\author{
S. AROCKIARAJ ${ }^{1}$, P. MAHALAKSHMI ${ }^{2}$, AND P. NAMASIVAYAM ${ }^{3}$
}

\begin{abstract}
An injective function $f: V(G) \rightarrow\{0,1,2, \ldots, q\}$ is an odd sum labeling if the induced edge labeling $f^{*}$ defined by $f^{*}(u v)=f(u)+f(v)$, for all $u v \in E(G)$, is bijective and $f^{*}(E(G))=\{1,3,5, \ldots, 2 q-1\}$. A graph is said to be an odd sum graph if it admits an odd sum labeling. In this paper, we have studied the odd sum property of the subdivision of the triangular snake, quadrilateral snake, slanting ladder, $C_{p} \odot K_{1}, H \odot K_{1}, C_{m} @ C_{n}$, the grid graph $P_{m} \times P_{n}$, duplication of a vertex of a path and duplication of a vertex of a cycle.
\end{abstract}

\section{INTRODUCTION}

Throughout this paper, by a graph, we mean a finite, undirected simple graph. Let $G(V, E)$ be a graph with $p$ vertices and $q$ edges. For notations and terminology, we follow [1].

A path on $p$ vertices is denoted by $P_{p}$ and a cycle on $p$ vertices is denoted by $C_{p}$. If $m$ number of pendant vertices are attached at each vertex of $G$, then the resultant graph obtained from $G$ is the graph $G \odot m K_{1}$. When $m=1, G \odot K_{1}$ is the corona of $G$. A triangular (quadrilateral) snake is obtained from a path by identifying each edge of the path with an edge of the cycle $C_{3}\left(C_{4}\right)$. The graph $C_{m} @ C_{n}$ is obtained by identifying an edge of $C_{m}$ with an edge of $C_{n}$. A graph which can be obtained from a given graph by breaking up each edge into one or more segments by inserting intermediate vertices between its two ends. If each edge of a graph $G$ is broken into two by exactly one vertex, then the resultant graph is taken as $S(G)$. The slanting ladder $S L_{n}$ is a graph obtained from two paths $u_{1} u_{2} \ldots u_{n}$ and $v_{1} v_{2} \ldots v_{n}$ by joining each $u_{i}$ with $v_{i+1}, 1 \leq i \leq n-1$.

Key words and phrases. Odd sum labeling, Odd sum graphs.

2010 Mathematics Subject Classification. $05 \mathrm{C} 78$.

Received: August 3, 2013

Revised: April 15, 2014. 
Duplication of a vertex $v$ of graph $G$ produces a new graph $G^{\prime}$ by adding a new vertex $v^{\prime}$ such that $N\left(v^{\prime}\right)=N(v)$. In other words, a vertex $v^{\prime}$ is said to be duplication of $v$ if all the vertices which are adjacent to $v$ in $G$ are also adjacent to $v^{\prime}$ in $G^{\prime}$.

In [2], an odd edge labeling of a graph is defined as follows: A labeling $f: V(G) \rightarrow$ $\{0,1,2, \ldots, p-1\}$ is called an odd edge labeling of $G$ if for the edge labeling $f^{+}$on $E(G)$ defined by $f^{+}(u v)=f(u)+f(v)$ for any edge $u v \in E(G)$, for a connected graph $G$, the edge labeling is not necessarily injective. In [5], the concept of pair sum labeling was introduced. An injective function $f: V(G) \rightarrow\{ \pm 1, \pm 2, \ldots, \pm p\}$ is said to be a pair sum labeling if the induced edge function $f_{e}: E(G) \rightarrow \mathcal{Z}-\{0\}$ defined by $f_{e}(u v)=f(u)+f(v)$ is one-one and $f_{e}(E(G))$ is either of the form $\left\{ \pm k_{1}, \pm k_{2}, \ldots, \pm \frac{k_{q}}{2}\right\}$ or $\left\{ \pm k_{1}, \pm k_{2}, \ldots, \pm \frac{k_{q-1}}{2}\right\} \cup\left\{\frac{k_{q+1}}{2}\right\}$ according as $q$ is even or odd. A graph with a pair sum labeling defined on it is called a pair sum graph. In [6], the concept of mean labeling was introduced. An injective function $f: V(G) \rightarrow\{0,1,2, \ldots, q\}$ is said to be a mean labeling if the induced edge labeling $f^{*}$ defined by

$$
f^{*}(u v)= \begin{cases}\frac{f(u)+f(v)}{2}, & \text { if } f(u)+f(v) \text { is even } \\ \frac{f(u)+f(v)+1}{2}, & \text { if } f(u)+f(v) \text { is odd }\end{cases}
$$

is injective and $f^{*}(E(G))=\{1,2, \ldots, q\}$. A graph $G$ is said to be odd mean graph if there exists an injective function $f$ from $V(G)$ to $\{0,1,2,3, \ldots, 2 q-1\}$ such that the induced map $f^{*}$ from $E(G) \rightarrow\{1,3,5, \ldots, 2 q-1\}$ defined by

$$
f^{*}(u v)= \begin{cases}\frac{f(u)+f(v)}{2}, & \text { if } f(u)+f(v) \text { is even } \\ \frac{f(u)+f(v)+1}{2}, & \text { if } f(u)+f(v) \text { is odd }\end{cases}
$$

is a bijection [4].

Motivated by these, we introduce a new concept called odd sum labeling. An injective function $f: V(G) \rightarrow\{0,1,2, \ldots, q\}$ is an odd sum labeling if the induced edge labeling $f^{*}$ defined by $f^{*}(u v)=f(u)+f(v)$, for all $u v \in E(G)$ is bijective and $f^{*}(E(G))=\{1,3,5, \ldots, 2 q-1\}$. A graph is said to be an odd sum graph if it admits an odd sum labeling.

In this paper, we have studied the odd sum property of the subdivision of the triangular snake, quadrilateral snake, slanting ladder, $C_{p} \odot K_{1}, H \odot K_{1}, C_{m} @ C_{n}$, the grid graph $P_{m} \times P_{n}$, duplication of a vertex of path and cycle by a vertex.

\section{Main Results}

Proposition 2.1. $S\left(T_{n}\right)$ is an odd sum graph, when $n$ is even.

Proof. Let $u_{1}, u_{2}, \ldots, u_{n}, u_{n+1}$ the vertices on the path of length $n$ in $T_{n}$ and let $v_{i}, 1 \leq i \leq n$ be the vertices of $T_{n}$ in which $v_{i}$ is adjacent to $u_{i}$ and $u_{i+1}$. Let $x_{i}, y_{i}$ and $z_{i}$ be the vertices which subdivide the edges $u_{i} u_{i+1}, u_{i} v_{i}$ and $v_{i} u_{i+1}$ respectively for each $i, 1 \leq i \leq n$. 
Define $f: V\left(S\left(T_{n}\right)\right) \rightarrow\{0,1,2, \ldots, 6 n\}$ as follows:

$$
\begin{aligned}
f\left(u_{i}\right) & =6 i-6, \quad 1 \leq i \leq n+1 \\
f\left(v_{i}\right) & = \begin{cases}6 i-4, & 1 \leq i \leq n \text { and } i \text { is odd } \\
6 i-2, & 1 \leq i \leq n \text { and } i \text { is even }\end{cases} \\
f\left(x_{i}\right) & = \begin{cases}6 i+1, & 1 \leq i \leq n \text { and } i \text { is odd } \\
6 i-7, & 1 \leq i \leq n \text { and } i \text { is even }\end{cases} \\
f\left(y_{i}\right) & = \begin{cases}6 i-5, & 1 \leq i \leq n \text { and } i \text { is odd } \\
6 i-3, & 1 \leq i \leq n \text { and } i \text { is even }\end{cases} \\
\text { and } f\left(z_{i}\right) & = \begin{cases}6 i-3, & 1 \leq i \leq n \text { and } i \text { is odd } \\
6 i-1, & 1 \leq i \leq n \text { and } i \text { is even. }\end{cases}
\end{aligned}
$$

Then the induced edge labeling is obtained as follows:

$$
\begin{aligned}
& f^{*}\left(u_{i} x_{i}\right)= \begin{cases}12 i-5, & 1 \leq i \leq n \text { and } i \text { is odd } \\
12 i-13, & 1 \leq i \leq n \text { and } i \text { is even }\end{cases} \\
& f^{*}\left(x_{i} u_{i+1}\right)= \begin{cases}12 i+1, & 1 \leq i \leq n \text { and } i \text { is odd } \\
12 i-7, & 1 \leq i \leq n \text { and } i \text { is even }\end{cases} \\
& f^{*}\left(u_{i} y_{i}\right)= \begin{cases}12 i-11, & 1 \leq i \leq n \text { and } i \text { is odd } \\
12 i-9, & 1 \leq i \leq n \text { and } i \text { is even }\end{cases} \\
& f^{*}\left(y_{i} v_{i}\right)= \begin{cases}12 i-9, & 1 \leq i \leq n \text { and } i \text { is odd } \\
12 i-5, & 1 \leq i \leq n \text { and } i \text { is even }\end{cases} \\
& f^{*}\left(v_{i} z_{i}\right)= \begin{cases}12 i-7, & 1 \leq i \leq n \text { and } i \text { is odd } \\
12 i-3, & 1 \leq i \leq n \text { and } i \text { is even }\end{cases} \\
& \text { and } f^{*}\left(z_{i} u_{i+1}\right)= \begin{cases}12 i-3, & 1 \leq i \leq n \text { and } i \text { is odd } \\
12 i-1, & 1 \leq i \leq n \text { and } i \text { is even. }\end{cases}
\end{aligned}
$$

Thus, $f$ is an odd sum labeling of $S\left(T_{n}\right)$ where $n$ is even.

Proposition 2.2. The graph $S\left(Q_{n}\right)$ is an odd sum graph for any $n$.

Proof. Let $u_{1}, u_{2}, \ldots, u_{n}, u_{n+1}$ be the vertices on the path of length $n$ in $Q_{n}$ and let $v_{i}$ and $w_{i}$ be the vertices of $Q_{n}$ in which $v_{i}$ is adjacent to $u_{i}$ and $w_{i}$ is adjacent to $u_{i+1}$, for each $i, 1 \leq i \leq n$. Let $t_{i}, x_{i}, y_{i}, z_{i}$ be the vertices which subdivide the edges $u_{i} u_{i+1}, u_{i} v_{i}, v_{i} w_{i}$ and $w_{i} u_{i+1}$ respectively for each $i, 1 \leq i \leq n$.

We define $f: V\left(S\left(Q_{n}\right)\right) \rightarrow\{0,1,2, \ldots, 8 n\}$ as follows: $f\left(u_{i}\right)=8 i-8,1 \leq i \leq n+1$, $f\left(v_{i}\right)=8 i-6,1 \leq i \leq n f\left(w_{i}\right)=8 i-2,1 \leq i \leq n, f\left(t_{i}\right)=8 i-1,1 \leq i \leq n$, $f\left(x_{i}\right)=8 i-7,1 \leq i \leq n f\left(y_{i}\right)=8 i-5,1 \leq i \leq n$ and $f\left(z_{i}\right)=8 i-3,1 \leq i \leq n$. Then the induced edge labeling is obtained as follows: $f^{*}\left(u_{i} x_{i}\right)=16 i-15,1 \leq i \leq n$, $f^{*}\left(x_{i} v_{i}\right)=16 i-13,1 \leq i \leq n, f^{*}\left(v_{i} y_{i}\right)=16 i-11,1 \leq i \leq n, f^{*}\left(y_{i} w_{i}\right)=16 i-7$, $1 \leq i \leq n, f^{*}\left(w_{i} z_{i}\right)=16 i-5,1 \leq i \leq n, f^{*}\left(z_{i} u_{i+1}\right)=16 i-3,1 \leq i \leq n$, $f^{*}\left(u_{i} t_{i}\right)=16 i-9,1 \leq i \leq n$ and $f^{*}\left(t_{i} u_{i+1}\right)=16 i-1,1 \leq i \leq n$.

Thus, $f$ is an odd sum labeling of $S\left(Q_{n}\right)$. 
Proposition 2.3. The subdivision graph of slanting ladder $S\left(S L_{n}\right)$ is an odd sum graph.

Proof. Let $u_{1}, u_{2}, \ldots, u_{n}$ and $v_{1}, v_{2}, \ldots, v_{n}$ be the vertices on the paths of length $n-$ 1. Let $x_{i}, y_{i}$ and $z_{i}$ be the vertices subdivided the edges $u_{i} u_{i+1}, v_{i} v_{i+1}$ and $u_{i} v_{i+1}$ respectively for each $i, 1 \leq i \leq n-1$.

Case (i). $\quad n$ is even.

We define $f: V\left(S\left(S L_{n}\right)\right) \rightarrow\{1,2, \ldots, 6(n-1)\}$ as follows:

$$
\begin{aligned}
& f\left(u_{i}\right)= \begin{cases}6 i-2, & 1 \leq i \leq n \text { and } i \text { is odd } \\
6 i-4, & 1 \leq i \leq n-2 \text { and } i \text { is even } \\
6(n-1), & i=n\end{cases} \\
& f\left(v_{i}\right)= \begin{cases}0, & i=1 \\
6 i-10, & 2 \leq i \leq n \text { and } i \text { is even } \\
6 i-8, & 2 \leq i \leq n \text { and } i \text { is odd }\end{cases} \\
& f\left(x_{i}\right)= \begin{cases}6 i-1, & 1 \leq i \leq n-1 \text { and } i \text { is odd } \\
6 i+3, & 1 \leq i \leq n-1 \text { and } i \text { is even }\end{cases} \\
& f\left(y_{i}\right)= \begin{cases}1, & i=1 \\
6 i-3, & 2 \leq i \leq n-1 \text { and } i \text { is even and } \\
6 i-7, & 2 \leq i \leq n-1 \text { and } i \text { is odd }\end{cases} \\
& f\left(z_{i}\right)= \begin{cases}3, & i=1 \\
6 i-5, & 2 \leq i \leq n-1 .\end{cases}
\end{aligned}
$$

Then the induced edge labeling is obtained as follows:

$$
\begin{aligned}
f^{*}\left(u_{i} x_{i}\right) & = \begin{cases}12 i-13, & 1 \leq i \leq n-1 \text { and } i \text { is odd } \\
12 i-1, & 1 \leq i \leq n-1 \text { and } i \text { is even }\end{cases} \\
f^{*}\left(x_{i} u_{i+1}\right) & = \begin{cases}12 i+1, & 1 \leq i \leq n-2 \text { and } i \text { is odd } \\
12 i+7, & 1 \leq i \leq n-2 \text { and } i \text { is even } \\
12 n-13, & i=n-1\end{cases} \\
f^{*}\left(v_{i} y_{i}\right) & = \begin{cases}1, & i=1 \\
12 i-13, & 2 \leq i \leq n-1 \text { and } i \text { is even } \\
12 i-15, & 2 \leq i \leq n-1 \text { and } i \text { is odd }\end{cases} \\
f^{*}\left(y_{i} v_{i+1}\right) & = \begin{cases}3, & i=1 \\
12 i-15, & 2 \leq i \leq n-1 \text { and } i \text { is even } \\
12 i-11, & 2 \leq i \leq n-1 \text { and } i \text { is odd }\end{cases} \\
f^{*}\left(u_{i} z_{i}\right) & = \begin{cases}7, & i=1 \\
12 i-9, & 2 \leq i \leq n-1 \text { and } i \text { is even } \\
12 i-7, & 2 \leq i \leq n-1 \text { and } i \text { is odd }\end{cases}
\end{aligned}
$$




$$
\text { and } f^{*}\left(z_{i} v_{i+1}\right)= \begin{cases}5, & i=1 \\ 12 i-9, & 2 \leq i \leq n-1 \text { and } i \text { is odd } \\ 12 i-7, & 2 \leq i \leq n-1 \text { and } i \text { is even }\end{cases}
$$

Thus, $f$ is an odd sum labeling of $S\left(S L_{n}\right)$, for $n \geq 4$.

When $n=2$, an odd sum labeling of the graph is given below.

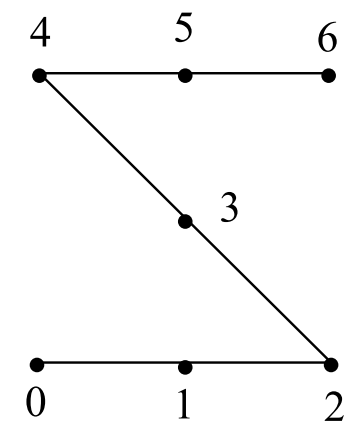

Figure 1. $S\left(S L_{2}\right)$

Case (ii). $n$ is odd.

We define $f: V\left(S L_{n}\right) \rightarrow\{1,2, \ldots, 6(n-1)\}$ as follows:

$$
\begin{aligned}
& f\left(u_{i}\right)= \begin{cases}4, & i=1 \\
12, & i=2 \\
6 i-4, & 3 \leq i \leq n-1 \text { and } i \text { is odd } \\
6 i-2, & 3 \leq i \leq n-1 \text { and } i \text { is even } \\
6(n-1), & i=n\end{cases} \\
& f\left(v_{i}\right)= \begin{cases}0, & i=1 \\
2, & i=2 \\
6, & i=3 \\
6 i-8, & 4 \leq i \leq n \text { and } i \text { is even } \\
6 i-10, & 4 \leq i \leq n \text { and } i \text { is odd }\end{cases} \\
& f\left(x_{i}\right)= \begin{cases}5, & i=1 \\
6 i-1, & 2 \leq i \leq n-1 \text { and } i \text { is even } \\
6 i+3, & 2 \leq i \leq n-1 \text { and } i \text { is odd }\end{cases} \\
& \text { and } f\left(z_{i}\right)= \begin{cases}1, & i=1 \\
9, & i=2 \\
6 i-3, & 3 \leq i \leq n-1 \text { and } i \text { is odd } \\
6 i-7, & 3 \leq i \leq n-1 \text { and } i \text { is even }\end{cases} \\
& \begin{array}{ll}
3, & i=1
\end{array}
\end{aligned}
$$


The induced edge labeling is obtained as follows:

$$
\begin{aligned}
& f^{*}\left(u_{i} x_{i}\right)= \begin{cases}9, & i=1 \\
23, & i=2 \\
12 i-1, & 3 \leq i \leq n-1 \text { and } i \text { is odd } \\
12 i-3, & 3 \leq i \leq n-1 \text { and } i \text { is even }\end{cases} \\
& f^{*}\left(x_{i} u_{i+1}\right)= \begin{cases}17, & i=1 \\
12 i+1, & 2 \leq i \leq n-2 \text { and } i \text { is even } \\
12 i+7, & 2 \leq i \leq n-2 \text { and } i \text { is odd } \\
12 n-13, & i=n-1\end{cases} \\
& f^{*}\left(v_{i} y_{i}\right)= \begin{cases}1, & i=1 \\
11, & i=2 \\
21, & i=3 \\
12 i-15, & 4 \leq i \leq n-1 \text { and } i \text { is even } \\
12 i-13, & 4 \leq i \leq n-1 \text { and } i \text { is odd }\end{cases} \\
& \text { and } f^{*}\left(z_{i} v_{i+1}\right)= \begin{cases}3, & i=1 \\
15, & i=2 \\
12 i-5, & 3 \leq i \leq n-1 \text { and } i \text { is odd } \\
12 i-11, & 3 \leq i \leq n-1 \text { and } i \text { is even } \\
12 i-7, & 3 \leq i \leq n-1 \text { and } i \text { is odd } \\
12 i-9, & 3 \leq i \leq n-1 \text { and } i \text { is even. }\end{cases} \\
& f^{*}\left(y_{i} v_{i+1}\right)= \begin{cases}7, & i=1 \\
19, & i=2 \\
12 i-9, & 3 \leq i \leq n-1 \text { and } i \text { is odd } \\
12 i-7, & 3 \leq i \leq n-1 \text { and } i \text { is even } \\
5, & i=1\end{cases}
\end{aligned}
$$

Thus, $f$ is an odd sum labeling for $n \geq 5$.

When $n=3$, an odd sum labeling of the graph is given below. 


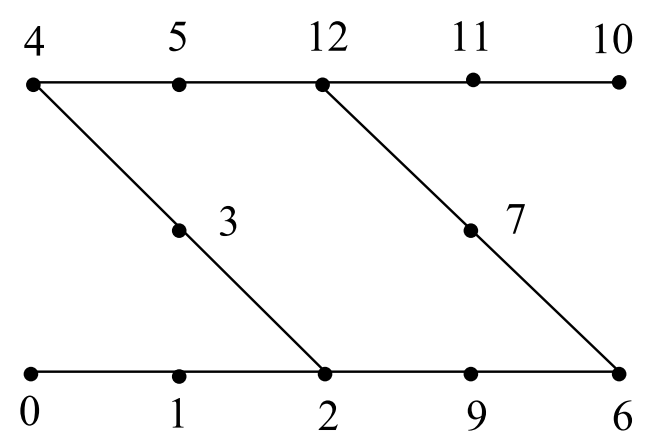

FIGURE 2

Hence, $S\left(S L_{n}\right)$ is an odd sum graph.

Proposition 2.4. The graph $S\left(C_{p} \odot K_{1}\right)$ is an odd sum graph.

Proof. Let $u_{1}, v_{1}, u_{2}, v_{2}, \ldots, u_{p}$ and $v_{p}$ be the vertices on the cycle and $u_{i} y_{i} x_{i}$ be the path on 3 vertices attached at each $u_{i}$.

Case (i). $p$ is even.

Define $f: V\left(S\left(C_{p} \odot K_{1}\right)\right) \rightarrow\{0,1,2, \ldots, 4 p\}$ as follows:

$$
\begin{aligned}
& f\left(u_{i}\right)= \begin{cases}4 i-3, & 1 \leq i \leq p \text { and } i \text { is odd } \\
4 i-1, & 1 \leq i \leq p \text { and } i \text { is even }\end{cases} \\
& f\left(v_{i}\right)= \begin{cases}4 i+2, & 1 \leq i \leq \frac{p}{2}-1 \text { and } i \text { is odd } \\
4 i, & 1 \leq i \leq \frac{p}{2}-1 \text { and } i \text { is even } \\
4 i+4, & \frac{p}{2} \leq i \leq p-1 \text { and } i \text { is odd } \\
4 i+2, & \frac{p}{2} \leq i \leq p-1 \text { and } i \text { is even } \\
0, & i=p\end{cases} \\
& f\left(x_{i}\right)= \begin{cases}4 i-1, & 1 \leq i \leq p \text { and } i \text { is odd } \\
4 i-3, & 1 \leq i \leq p \text { and } i \text { is even }\end{cases} \\
& f\left(y_{i}\right)= \begin{cases}4 i-2, & 1 \leq i \leq \frac{p}{2} \text { and } i \text { is odd } \\
4 i-4, & 1 \leq i \leq \frac{p}{2} \text { and } i \text { is even } \\
4 i, & \frac{p}{2}+1 \leq i \leq p-1 \text { and } i \text { is odd } \\
4 i-2, & \frac{p}{2}+1 \leq i \leq p-1 \text { and } i \text { is even } \\
0, & i=p .\end{cases}
\end{aligned}
$$

Then the induced edge labeling is obtained as follows:

$$
f^{*}\left(u_{i} v_{i}\right)= \begin{cases}8 i-1, & 1 \leq i \leq \frac{p}{2}-1 \\ 8 i+1, & \underline{p} 2 \leq i \leq p-1 \\ 4 p-1, & i=p\end{cases}
$$




$$
\begin{aligned}
& f^{*}\left(v_{i} u_{i+1}\right)= \begin{cases}8 i+5, & 1 \leq i \leq \frac{p}{2}-1 \text { and } i \text { is odd } \\
8 i+1, & 1 \leq i \leq \frac{p}{2}-1 \text { and } i \text { is even } \\
8 i+7, & \frac{p}{2} \leq i \leq p-1 \text { and } i \text { is odd } \\
8 i+3, & \frac{p}{2} \leq i \leq p-1 \text { and } i \text { is even }\end{cases} \\
& f^{*}\left(u_{i} y_{i}\right)= \begin{cases}8 i-5, & 1 \leq i \leq \frac{p}{2} \\
8 i-3, & \frac{p}{2}+1 \leq i \leq p\end{cases} \\
& f^{*}\left(y_{i} x_{i}\right)= \begin{cases}8 i-3, & 1 \leq i \leq \frac{p}{2} \text { and } i \text { is odd } \\
8 i-7, & 1 \leq i \leq \frac{p}{2} \text { and } i \text { is even } \\
8 i-1, & \frac{p}{2}+1 \leq i \leq p \text { and } i \text { is odd } \\
8 i-5, & \frac{p}{2}+1 \leq i \leq p \text { and } i \text { is even }\end{cases}
\end{aligned}
$$

and $f^{*}\left(v_{p} u_{1}\right)=1$.

Thus, $f$ is an odd sum labeling of $S\left(C_{p} \odot K_{1}\right)$.

Case (ii). $\quad p$ is odd, $p \equiv 1(\bmod 4)$.

The labeling $f: V\left(S\left(C_{p} \odot K_{1}\right)\right) \rightarrow\{0,1,2, \ldots, 4 p\}$ is defined as follows:

$$
\begin{aligned}
f\left(u_{i}\right)= \begin{cases}4 i-2, & 1 \leq i \leq p \text { and } i \text { is even } \\
4 i-4, & 1 \leq i \leq \frac{p-3}{2} \text { and } i \text { is odd } \\
4 i, & \frac{p-1}{2} \leq i \leq p-1 \text { and } i \text { is odd } \\
4 p-2, & i=p\end{cases} \\
f\left(x_{i}\right)= \begin{cases}4 i-4, & 1 \leq i \leq \frac{p-1}{2} \text { and } i \text { is even } \\
4 i, & \frac{p+3}{2} \leq i \leq p \text { and } i \text { is even } \\
4 i-2, & 1 \leq i \leq \frac{p-3}{2} \text { and } i \text { is odd } \\
4 i-4, & i=\frac{p+1}{2} \\
4 i-2, & \frac{p+3}{2} \leq i \leq p-1 \text { and } i \text { is odd } \\
4 p, & i=p\end{cases} \\
f\left(y_{i}\right)= \begin{cases}4 i-5, & 1 \leq i \leq \frac{p-1}{2} \text { and } i \text { is even } \\
4 i-3, & \frac{p+3}{2} \leq i \leq p \text { and } i \text { is even } \\
4 i-3, & 1 \leq i \leq \frac{p+1}{2} \text { and } i \text { is odd } \\
4 i-5, & \frac{p+3}{2} \leq i \leq p-1 \text { and } i \text { is odd } \\
4 p-1, & i=p\end{cases} \\
\text { and } f\left(v_{i}\right)= \begin{cases}4 i+1, & 1 \leq i \leq \frac{p-3}{2} \text { and } i \text { is odd } \\
4 i-1, & \frac{p+1}{2} \leq i \leq p-2 \text { and } i \text { is odd } \\
4 i-1, & 1 \leq i \leq \frac{p-1}{2} \text { and } i \text { is even } \\
4 i+1, & \frac{p+3}{2} \leq i \leq p-1 \text { and } i \text { is even } \\
4 p-5, & i=p\end{cases}
\end{aligned}
$$

The induced edge labeling is obtained as follows:

$$
f^{*}\left(u_{i} v_{i}\right)= \begin{cases}8 i-3, & 1 \leq i \leq \frac{p-1}{2} \\ 8 i-1, & \frac{p+1}{2} \leq i \leq p-1 \\ 8 p-7, & i=p\end{cases}
$$




$$
\begin{aligned}
& f^{*}\left(v_{i} u_{i+1}\right)= \begin{cases}8 i+3, & 1 \leq i \leq \frac{p-3}{2} \text { and } i \text { is odd } \\
8 i+1, & \frac{p+1}{2} \leq i \leq p-2 \text { and } i \text { is odd } \\
8 i-1, & 1 \leq i \leq \frac{p-5}{2} \text { and } i \text { is even } \\
8 i+3, & i=\frac{p-1}{2} \\
8 i+5, & \frac{p+3}{2} \leq i \leq p-3 \text { and } i \text { is even } \\
8 p-5, & i=p-1\end{cases} \\
& f^{*}\left(v_{p} u_{1}\right)=4 p-5
\end{aligned}
$$

Thus, $f$ is an odd sum labeling of $S\left(C_{p} \odot K_{1}\right)$.

Case (iii). $\quad p$ is odd and $p \equiv 3(\bmod 4)$.

The labeling $f$ is defined as follows:

$$
\begin{aligned}
f\left(u_{i}\right)= \begin{cases}4 i-4, & 1 \leq i \leq \frac{p-1}{2} \text { and } i \text { is odd } \\
4 i-2, & \frac{p+3}{2} \leq i \leq p \text { and } i \text { is odd } \\
4 i-2, & 1 \leq i \leq \frac{p-3}{2} \text { and } i \text { is even } \\
4 i, & \frac{p+1}{2} \leq i \leq p \text { and } i \text { is even }\end{cases} \\
f\left(v_{i}\right)= \begin{cases}4 i+1, & 1 \leq i \leq p-2 \text { and } i \text { is odd } \\
4 p-3, & i=p \\
4 i+3, & 1 \leq i \leq p-1 \text { and } i \text { is even }\end{cases} \\
f\left(x_{i}\right)= \begin{cases}4 i-2, & 1 \leq i \leq \frac{p-1}{2} \text { and } i \text { is odd } \\
4 i, & \frac{p+3}{2} \leq i \leq p \text { and } i \text { is odd } \\
4 i-4, & 1 \leq i \leq \frac{p+1}{2} \text { and } i \text { is even } \\
4 i-2, & \frac{p+5}{2} \leq i \leq p \text { and } i \text { is even }\end{cases} \\
\text { and } f\left(y_{i}\right)= \begin{cases}4 i-3, & 1 \leq i \leq p-2 \text { and } i \text { is odd } \\
4 p-1, & i=p \\
4 i-5, & 1 \leq i \leq p-1 \text { and } i \text { is even. }\end{cases}
\end{aligned}
$$

The induced edge labeling is obtained as follows:

$$
f^{*}\left(u_{i} v_{i}\right)= \begin{cases}8 i-3, & 1 \leq i \leq \frac{p-1}{2} \\ 8 i-1, & \frac{p+1}{2} \leq i \leq p-1 \\ 8 p-5, & i=p\end{cases}
$$




$$
\begin{aligned}
& f^{*}\left(v_{i} u_{i+1}\right)= \begin{cases}8 i+3, & 1 \leq i \leq \frac{p-5}{2} \text { and } i \text { is odd } \\
8 i+5, & \frac{p-1}{2} \leq i \leq p-2 \text { and } i \text { is odd } \\
8 i-1, & 1 \leq i \leq \frac{p-3}{2} \text { and } i \text { is even } \\
8 i+1, & \frac{p+1}{2} \leq i \leq p-1 \text { and } i \text { is even }\end{cases} \\
& f^{*}\left(v_{p} u_{1}\right)=4 p-3 \\
& f^{*}\left(u_{i} y_{i}\right)= \begin{cases}8 i-7, & 1 \leq i \leq \frac{p-1}{2} \\
8 i-5, & \frac{p+1}{2} \leq i \leq p-1 \\
8 p-3, & i=p\end{cases} \\
& \text { and } f^{*}\left(y_{i} x_{i}\right)= \begin{cases}8 i-5, & 1 \leq i \leq \frac{p-1}{2} \text { and } i \text { is odd } \\
8 i-3, & \frac{p+3}{2} \leq i \leq p-1 \text { and } i \text { is odd } \\
8 p-1, & i=p \\
8 i-9, & 1 \leq i \leq \frac{p+1}{2} \text { and } i \text { is even } \\
8 i-7, & \frac{p+5}{2} \leq i \leq p-1 \text { and } i \text { is even. }\end{cases}
\end{aligned}
$$

Hence, $f$ is an odd sum labeling of $S\left(C_{p} \odot K_{1}\right)$.

Proposition 2.5. The graph $S\left(H_{n} \odot K_{1}\right)$ is an odd sum graph.

Proof. Let $u_{1}, u_{2}, \ldots, u_{n}$ and $v_{1}, v_{2}, \ldots, v_{n}$ be the vertices of the paths of length $n-1$. Let $a_{1, i} a_{2, i} u_{i}$ be the path attached at each $u_{i}, 1 \leq i \leq n$ and $b_{1, i} b_{2, i} v_{i}$ be the path attached at each $v_{i}, 1 \leq i \leq n$. Each edge $u_{i} u_{i+1}$ is subdivided by a vertex $x_{i}, 1 \leq$ $i \leq n-1$ and each edge $v_{i} v_{i+1}$ is subdivided by a vertex $y_{i}, 1 \leq i \leq n-1$. The edge $u_{\frac{n+1}{2}} v_{\frac{n+1}{2}}$ is divided by a vertex $z$ when $n$ is odd. The edge $u_{\frac{n+2}{2}} v_{\frac{n}{2}}$ is divided by a vertex $z$ when $n$ is even.

Case (i). $n$ is odd.

The labeling $f: V\left(S\left(H_{n} \odot K_{1}\right)\right) \rightarrow\{0,1,2, \ldots, 8 n-2\}$ is defined as follows:

$$
\begin{aligned}
f\left(u_{i}\right) & = \begin{cases}4 i-2, & 1 \leq i \leq n \text { and } i \text { is odd } \\
4 i-4, & 1 \leq i \leq n \text { and } i \text { is even }\end{cases} \\
f\left(v_{i}\right) & = \begin{cases}4(n+i)-4, & 1 \leq i \leq n \text { and } i \text { is odd } \\
4(n+i)-2, & 1 \leq i \leq n \text { and } i \text { is even }\end{cases} \\
f\left(a_{1, i}\right) & = \begin{cases}4 i-4, & 1 \leq i \leq n \text { and } i \text { is odd } \\
4 i-2, & 1 \leq i \leq n \text { and } i \text { is even }\end{cases} \\
f\left(a_{2, i}\right) & = \begin{cases}1, & i=1 \\
4 i-5, & 2 \leq i \leq n \text { and } i \text { is odd } \\
4 i-3, & 1 \leq i \leq n \text { and } i \text { is even }\end{cases} \\
f\left(b_{1, i}\right) & = \begin{cases}4(n+i)-2, & 1 \leq i \leq n \text { and } i \text { is odd } \\
4(n+i)-4, & 1 \leq i \leq n \text { and } i \text { is even }\end{cases}
\end{aligned}
$$




$$
\begin{aligned}
& f\left(b_{2, i}\right)=\left\{\begin{array}{l}
4(n+i)-5, \quad 1 \leq i \leq \frac{n-1}{2} \text { and } i \text { is odd } \\
4(n+i)-3, \quad \frac{n+1}{2} \leq i \leq n \text { and } i \text { is odd } \\
4(n+i)-7, \quad 1 \leq i \leq \frac{n-1}{2} \text { and } i \text { is even } \\
4(n+i)-5, \quad \frac{n+1}{2} \leq i \leq n \text { and } i \text { is even }
\end{array}\right. \\
& f\left(x_{i}\right)= \begin{cases}4 i-1, & 1 \leq i \leq n-1 \text { and } i \text { is odd } \\
4 i+1, & 1 \leq i \leq n-1 \text { and } i \text { is even }\end{cases} \\
& f\left(y_{i}\right)= \begin{cases}4(n+i)-1, & 1 \leq i \leq \frac{n-1}{2} \text { and } i \text { is odd } \\
4(n+i)+1, & \frac{n+1}{2} \leq i \leq n-1 \text { and } i \text { is odd } \\
4(n+i)-3, & 1 \leq i \leq \frac{n-1}{2} \text { and } i \text { is even } \\
4(n+i)-1, & \frac{n+1}{2} \leq i \leq n-1 \text { and } i \text { is even }\end{cases}
\end{aligned}
$$

and $f(z)=6 n-3$.

The induced edge labeling is obtained as follows:

$$
\begin{aligned}
& f^{*}\left(u_{i} x_{i}\right)=8 i-3, \quad 1 \leq i \leq n-1 \\
& f^{*}\left(x_{i} u_{i+1}\right)= \begin{cases}8 i-1, & 1 \leq i \leq n-1 \text { and } i \text { is odd } \\
8 i+3, & 1 \leq i \leq n-1 \text { and } i \text { is even }\end{cases} \\
& f^{*}\left(v_{i} y_{i}\right)= \begin{cases}8(n+i)-5, & 1 \leq i \leq \frac{n-1}{2} \\
8(n+i)-3, & \frac{n+1}{2} \leq i \leq n-1\end{cases} \\
& f^{*}\left(y_{i} v_{i+1}\right)= \begin{cases}8(n+i)+1, & 1 \leq i \leq \frac{n-1}{2} \text { and } i \text { is odd } \\
8(n+i)+3, & \frac{n+1}{2} \leq i \leq n-1 \text { and } i \text { is odd } \\
8(n+i)-3, & 1 \leq i \leq \frac{n-1}{2} \text { and } i \text { is even } \\
8(n+i)-1, & \frac{n+1}{2} \leq i \leq n-1 \text { and } i \text { is even }\end{cases} \\
& f^{*}\left(a_{1, i} a_{2, i}\right)= \begin{cases}1, & i=1 \\
8 i-9, & 2 \leq i \leq n \text { and } i \text { is odd } \\
8 i-5, & 2 \leq i \leq n \text { and } i \text { is even }\end{cases} \\
& f^{*}\left(a_{2, i} u_{i}\right)= \begin{cases}3, & i=1 \\
8 i-7, & 2 \leq i \leq n\end{cases} \\
& f^{*}\left(b_{2, i} b_{1, i}\right)= \begin{cases}8(n+i)-7, & 1 \leq i \leq \frac{n-1}{2} \text { and } i \text { is odd } \\
8(n+i)-5, & \frac{n+1}{2} \leq i \leq n \text { and } i \text { is odd } \\
8(n+i)-11, & 1 \leq i \leq \frac{n-1}{2} \text { and } i \text { is even } \\
8(n+i)-9, & \frac{n+1}{2} \leq i \leq n \text { and } i \text { is even }\end{cases} \\
& f^{*}\left(v_{i} b_{2, i}\right)= \begin{cases}8(n+i)-9, & 1 \leq i \leq \frac{n-1}{2} \\
8(n+i)-7, & \frac{n+1}{2} \leq i \leq n\end{cases} \\
& f^{*}\left(u_{\frac{n+1}{2}} z\right)=8 n-3
\end{aligned}
$$

and $f^{*}\left(z v_{\frac{n+1}{2}}\right)=12 n-5$. 
Case (ii). $n$ is even.

The labeling $f: V\left(S\left(H_{n} \odot K_{1}\right)\right) \rightarrow\{0,1,2, \ldots, 8 n-2\}$ is defined as follows:

$$
\begin{aligned}
& f\left(u_{i}\right)= \begin{cases}4 i-2, & 1 \leq i \leq n \text { and } i \text { is odd } \\
4 i-4, & 1 \leq i \leq n \text { and } i \text { is even }\end{cases} \\
& f\left(a_{1, i}\right)= \begin{cases}4 i-4, & 1 \leq i \leq n \text { and } i \text { is odd } \\
4 i-2, & 1 \leq i \leq n \text { and } i \text { is even }\end{cases} \\
& f\left(a_{2, i}\right)= \begin{cases}1, & i=1 \\
4 i-5, & 2 \leq i \leq n \text { and } i \text { is odd } \\
4 i-3, & 1 \leq i \leq n \text { and } i \text { is even }\end{cases} \\
& f\left(v_{i}\right)= \begin{cases}4(n+i)-2, & 1 \leq i \leq n \text { and } i \text { is odd } \\
4(n+i)-4, & 1 \leq i \leq n \text { and } i \text { is even }\end{cases} \\
& f\left(b_{1, i}\right)= \begin{cases}4(n+i)-4, & 1 \leq i \leq n \text { and } i \text { is odd } \\
4(n+i)-2, & 1 \leq i \leq n \text { and } i \text { is even }\end{cases} \\
& f\left(b_{2, i}\right)= \begin{cases}4(n+i)-5, & i=1 \\
4(n+i)-7, & 2 \leq i \leq \frac{n}{2} \text { and } i \text { is odd } \\
4(n+i)-5, & \frac{n+2}{2} \leq i \leq n \text { and } i \text { is odd } \\
4(n+i)-5, & 2 \leq i \leq \frac{n-2}{2} \text { and } i \text { is even } \\
4(n+i)-3, & \frac{n}{2} \leq i \leq n \text { and } i \text { is even }\end{cases} \\
& \text { and } f(z)= \begin{cases}6 n-3, & n \equiv 2(\bmod 4) \\
6 n-5, & n \equiv 0(\bmod 4) .\end{cases} \\
& f\left(x_{i}\right)= \begin{cases}4-1, & 1 \leq i \leq n-1 \text { and } i \text { is odd } \\
4 i+1, & 1 \leq i \leq n \text { and } i \text { is even }\end{cases} \\
& \begin{array}{ll}
4(n+i)-3, & 1 \leq i \leq \frac{n-2}{2} \text { and } i \text { is odd } \\
4(n+i)-1, & \frac{n}{2} \leq i \leq n-1 \text { and } i \text { is odd } \\
4(n+i)-1, & 1 \leq i \leq \frac{n-2}{2} \text { and } i \text { is even } \\
4(n+i)+1, & \frac{n}{2} \leq i \leq n-1 \text { and } i \text { is even }
\end{array}
\end{aligned}
$$

The induced edge labeling is obtained as follows:

$$
\begin{aligned}
f^{*}\left(u_{i} x_{i}\right) & =8 i-3, \quad 1 \leq i \leq n-1 \\
f^{*}\left(x_{i} u_{i+1}\right) & = \begin{cases}8 i-1, & 1 \leq i \leq n-1 \text { and } i \text { is odd } \\
8 i+3, & 1 \leq i \leq n-1 \text { and } i \text { is even }\end{cases} \\
f^{*}\left(v_{i} y_{i}\right) & = \begin{cases}8(n+i)-5, & 1 \leq i \leq \frac{n-2}{2} \\
8(n+i)-3, & \frac{n}{2} \leq i \leq n-1\end{cases} \\
f^{*}\left(y_{i} v_{i+1}\right) & = \begin{cases}8(n+i)-3, & 1 \leq i \leq \frac{n-2}{2} \text { and } i \text { is odd } \\
8(n+i)-1, & \frac{n}{2} \leq i \leq n-1 \text { and } i \text { is odd } \\
8(n+i)+1, & 1 \leq i \leq \frac{n-2}{2} \text { and } i \text { is even } \\
8(n+i)+3, & \frac{n}{2} \leq i \leq n-1 \text { and } i \text { is even }\end{cases}
\end{aligned}
$$




$$
\begin{aligned}
& f^{*}\left(a_{1, i} a_{2, i}\right)= \begin{cases}1, & i=1 \\
8 i-9, & 2 \leq i \leq n \text { and } i \text { is odd } \\
8 i-5, & 2 \leq i \leq n \text { and } i \text { is even }\end{cases} \\
& f^{*}\left(a_{2, i} u_{i}\right)= \begin{cases}3, & i=1 \\
8 i-7, & 2 \leq i \leq n\end{cases} \\
& f^{*}\left(b_{2, i} b_{1, i}\right)= \begin{cases}8(n+i)-9, & i=1 \\
8(n+i)-11, & 2 \leq i \leq \frac{n}{2} \text { and } i \text { is odd } \\
8(n+i)-9, & \frac{n+2}{2} \leq i \leq n \text { and } i \text { is odd } \\
8(n+i)-7, & 2 \leq i \leq \frac{n-2}{2} \text { and } i \text { is even } \\
8(n+i)-5, & \frac{n}{2} \leq i \leq n \text { and } i \text { is even }\end{cases} \\
& f^{*}\left(v_{i} b_{2, i}\right)= \begin{cases}8(n+i)-7, & i=1 \\
8(n+i)-9, & 2 \leq i \leq \frac{n}{2} \text { and } i \text { is odd } \\
8(n+i)-7, & \frac{n+2}{2} \leq i \leq n \text { and } i \text { is odd } \\
8(n+i)-9, & 2 \leq i \leq \frac{n-2}{2} \text { and } i \text { is even } \\
8(n+i)-7, & \frac{n}{2} \leq i \leq n \text { and } i \text { is even }\end{cases} \\
& \text { and } f^{*}\left(z v_{\frac{n}{2}}\right)=\left\{\begin{array}{l}
12 n-5, \quad n \equiv 2(\bmod 4) \\
12 n-9, \quad n \equiv 0(\bmod 4) .
\end{array}\right.
\end{aligned}
$$

Hence, $f$ is an odd sum labeling.

Proposition 2.6. The graph $S\left(C_{m} @ C_{n}\right)$ is an odd sum graph for any positive integers $m, n \geq 3$.

Proof. In $S\left(C_{m} @ C_{n}\right), 2(m+n-2)$ vertices lies on the circle and one vertex lies on a chord. Let $v_{1}, v_{2}, \ldots, v_{2(m+n-2)}$ be the vertices on the cycle in $S\left(C_{m} @ C_{n}\right)$ and $v_{2(m+n-2)+1}$ be the vertex having neighbours $v_{2 n-2}$ and $v_{2(m+n-2)}$.

Case (i). $m$ and $n$ are odd with $n \geq m$.

$$
f\left(v_{i}\right)= \begin{cases}2 m+2 n-4, & i=1 \\ i-1, & 2 \leq i \leq m+2 n-4 \text { and } i \text { is even } \\ i+1, & m+2 n-2 \leq i \leq 2 m+2 n-4 \text { and } i \text { is even } \\ i-3, & 3 \leq i \leq m+n-1 \text { and } i \text { is odd } \\ i-1, & m+n-3 \leq i \leq 2 m+2 n-4 \text { and } i \text { is odd } \\ 2 m+2 n-2, & i=2 m+2 n-3 .\end{cases}
$$

The induced edge label is obtained as follows:

$$
\begin{aligned}
f^{*}\left(v_{i} v_{i+1}\right) & = \begin{cases}2 m+2 n-3, & i=1 \\
2 i-3, & 2 \leq i \leq m+n-1 \\
2 i-1, & m+n \leq i \leq m+2 n-3 \\
2 i+1, & m+2 n-2 \leq i \leq 2 m+2 n-5\end{cases} \\
f^{*}\left(v_{2 n-2} v_{2 m+2 n-3}\right) & =2 m+4 n-5 \text { and } \\
f^{*}\left(v_{2 m+2 n-4} v_{2 m+2 n-3}\right) & =4 m+4 n-5 .
\end{aligned}
$$


Case (ii). $\quad m$ is odd, $n$ is even and $n \geq m+3$.

$$
f\left(v_{i}\right)= \begin{cases}2 m+2 n-4, & i=1 \\ i-1, & 2 \leq i \leq m+n-1 \text { and } i \text { is even } \\ i+1, & m+n+1 \leq i \leq 2 m+2 n-4 \text { and } i \text { is even } \\ i-3, & 3 \leq i \leq m+2 n-2 \text { and } i \text { is odd } \\ i-1, & m+2 n-4 \leq i \leq 2 m+2 n-4 \text { and } i \text { is even } \\ 2 m+2 n-2, & i=2 m+2 n-3\end{cases}
$$

The induced edge label is obtained as follows:

$$
\begin{aligned}
f^{*}\left(v_{i} v_{i+1}\right) & = \begin{cases}2 m+2 n-3, & i=1 \\
2 i-3, & 2 \leq i \leq m+n-1 \\
2 i-1, & m+n \leq i \leq m+2 n-1 \\
2 i+1, & m+2 n \leq i \leq 2 m+2 n-5\end{cases} \\
f^{*}\left(v_{2 n-2} v_{2 m+2 n-3}\right) & =2 m+4 n-3 \text { and } \\
f^{*}\left(v_{2 m+2 n-4} v_{2 m+2 n-3}\right) & =4 m+4 n-5
\end{aligned}
$$

Case (iii). $\quad m$ is odd $n=m+1$.

$$
f\left(v_{i}\right)= \begin{cases}i, & 1 \leq i \leq 2 m+2 n-3 \text { and } i \text { is odd } \\ i-2, & 2 \leq i \leq 2 n-2 \text { and } i \text { is even } \\ i, & 2 n \leq i \leq m+2 n-5 \text { and } i \text { is even } \\ i+2, & m+2 n-4 \leq i \leq 2 m+2 n-4 \text { and } i \text { is even. }\end{cases}
$$

The induced edge label is obtained as follows:

$$
\begin{aligned}
f^{*}\left(v_{i} v_{i+1}\right) & = \begin{cases}2 i-1, & 1 \leq i \leq 2 n-2 \\
2 i+1, & 2 n-1 \leq i \leq m+2 n-5 \\
2 i+3, & m+2 n-4 \leq i \leq 2 m+2 n-5\end{cases} \\
f^{*}\left(v_{2 m+2 n-4} v_{1}\right) & =2 m+2 n-1 \\
f^{*}\left(v_{2 n-2} v_{2 m+2 n-3}\right) & =2 m+4 n-7 \text { and } \\
f^{*}\left(v_{2 m+2 n-4} v_{2 m+2 n-3}\right) & =4 m+4 n-5 .
\end{aligned}
$$

Case (iv). $m$ is even and $n \geq m+1$ is even.

$$
f\left(v_{i}\right)= \begin{cases}i-1, & 1 \leq i \leq m+n-2 \text { and } i \text { is odd } \\ i+1, & m+n \leq i \leq 2 m+2 n-3 \text { and } i \text { is odd } \\ i-1, & 2 \leq i \leq m+2 n-4 \text { and } i \text { is even } \\ i+1, & m+2 n-5 \leq i \leq 2 m+2 n-4 \text { and } i \text { is even. }\end{cases}
$$

The induced edge label is obtained as follows:

$$
f^{*}\left(v_{i} v_{i+1}\right)= \begin{cases}2 i-1, & 1 \leq i \leq m+n-2 \\ 2 i+1, & m+n-1 \leq i \leq m+2 n-4 \\ 2 i+3, & m+2 n-3 \leq i \leq 2 m+2 n-4\end{cases}
$$




$$
\begin{aligned}
f^{*}\left(v_{2 m+2 n-4} v_{1}\right) & =2 m+2 n-3 \text { and } \\
f^{*}\left(v_{2 n-2} v_{2 m+2 n-3}\right) & =2 m+4 n-5 .
\end{aligned}
$$

Case (v). $m$ is even and $n \geq m+2$ is even.

$$
f\left(v_{i}\right)= \begin{cases}i-1, & 1 \leq i \leq m+2 n-3 \text { and } i \text { is odd } \\ i+1, & m+2 n-4 \leq i \leq 2 m+2 n-3 \text { and } i \text { is odd } \\ i-1, & 2 \leq i \leq m+n-2 \text { and } i \text { is even } \\ i+1, & m+n-1 \leq i \leq 2 m+2 n-4 \text { and } i \text { is even. }\end{cases}
$$

The induced edge label is obtained as follows:

$$
\begin{aligned}
& f^{*}\left(v_{i} v_{i+1}\right)= \begin{cases}2 i-1, & 1 \leq i \leq m+n-2 \\
2 i+1, & m+n-1 \leq i \leq m+2 n-3 \\
2 i+3, & m+2 n-2 \leq i \leq 2 m+2 n-4\end{cases} \\
& f^{*}\left(v_{2 m+2 n-4} v_{1}\right)=2 m+2 n-3 \text { and } \\
& f^{*}\left(v_{2 n-2} v_{2 m+2 n-3}\right)=2 m+4 n-3 .
\end{aligned}
$$

Case (vi). $m$ is even and $n=m$.

$$
f\left(v_{i}\right)= \begin{cases}i-1, & 1 \leq i \leq m-1 \text { and } i \text { is odd } \\ i+1, & m \leq i \leq 2 m-3 \text { and } i \text { is odd } \\ 6 m-i-3, & 2 m-2 \leq i \leq 4 m-3 \text { and } i \text { is odd } \\ i-1, & 2 \leq i \leq 2 m-2 \text { and } i \text { is even } \\ 6 m-i-3, & 2 m-1 \leq i \leq 3 m-2 \text { and } i \text { is even } \\ 6 m-i-5, & 3 m-1 \leq i \leq 4 m-4 \text { and } i \text { is even. }\end{cases}
$$

The induced edge label is obtained as follows:

$$
\begin{aligned}
f^{*}\left(v_{i} v_{i+1}\right) & = \begin{cases}2 i-1, & 1 \leq i \leq m-1 \\
2 i+1, & m \leq i \leq 2 m-3 \\
6 m-5, & i=2 m-2 \\
12 m-2 i-7, & 2 m-1 \leq i \leq 3 m-2 \\
12 m-2 i-9, & 3 m-1 \leq i \leq 4 m-4\end{cases} \\
f^{*}\left(v_{4 m-4} v_{1}\right) & =2 m-1 \text { and } \\
f^{*}\left(v_{2 m-2} v_{4 m-3}\right) & =4 m-3 .
\end{aligned}
$$

Hence, $f$ is an odd sum labeling. Hence, $S\left(C_{m} @ C_{n}\right)$ is an odd sum graph.

Proposition 2.7. The Grid $S\left(P_{m} \times P_{n}\right)$ is an odd sum graph.

Proof. Let $u_{i, j}, 1 \leq i \leq m$ and $1 \leq j \leq n$ be the vertices of the grid $P_{m} \times P_{n}$. Let $v_{i, j}$ be the vertex divides the edge $u_{i, j} u_{i, j+1}$ for each $1 \leq i \leq m$ and $1 \leq j \leq n-1$ and $w_{i, j}$ be the vertex divides the edge $u_{i, j} u_{i+1, j}$ for each $1 \leq i \leq m-1$ and $1 \leq j \leq n$. 
We define $f: V\left(S\left(P_{m} \times P_{n}\right)\right) \rightarrow\{0,1,2, \ldots, 4 m n-2 m-2 n\}$ as follows:

$f\left(u_{i, j}\right)= \begin{cases}2(i-1)(2 n-1)+2 j-2, & 1 \leq i \leq m \text { and } i \text { is odd, } 1 \leq j \leq n \\ 2(i-2)(2 n-1)+6 n-2 j-2, & 1 \leq i \leq m \text { and } i \text { is even, } 1 \leq j \leq n\end{cases}$ $f\left(v_{i, j}\right)= \begin{cases}2 j-1, & i=1,1 \leq j \leq n-1 \\ (2 i-3)(2 n-1)+4 j-2, & 3 \leq i \leq m \text { and } i \text { is odd, } 1 \leq j \leq n-1 \\ (2 i-1)(2 n-1)-4 j, & 2 \leq i \leq m \text { and } i \text { is even, } 1 \leq j \leq n-1\end{cases}$ $f\left(w_{i, j}\right)= \begin{cases}(2 i+1)(2 n-1)-4 j+2, & 1 \leq i \leq m-1 \text { and } i \text { is odd, } 1 \leq j \leq n \\ (2 i-1)(2 n-1)+4(j-1), & 1 \leq i \leq m-1 \text { and } i \text { is even, } 1 \leq j \leq n .\end{cases}$

The induced edge label is obtained as follows:

$$
\begin{aligned}
& f^{*}\left(u_{i, j} v_{i, j}\right)= \begin{cases}4 j-3, & i=1 \text { and } 1 \leq j \leq n-1 \\
(4 i-5)(2 n-1)+6 j-4, & 3 \leq i \leq m \text { and } i \text { is odd } \\
(4 i-5)(2 n-1)+6 n-6 j-2, & 1 \leq j \leq n-1 \\
& 2 \leq i \leq m \text { and } i \text { is even } \\
& 1 \leq j \leq n-1\end{cases} \\
& f^{*}\left(v_{i, j} u_{i, j+1}\right)= \begin{cases}4 j-1, & i=1 \text { and } 1 \leq j \leq n-1 \\
(4 i-5)(2 n-1)+6 j-2, & 3 \leq i \leq m \text { and } i \text { is odd } \\
(4 i-5)(2 n-1)+6 n-6 j-4, & 1 \leq j \leq n-1 \\
& 2 \leq i \leq m \text { and } i \text { is even } \\
& 1 \leq j \leq n-1\end{cases} \\
& f^{*}\left(u_{i, j} w_{i, j}\right)= \begin{cases}(4 i-1)(2 n-1)-2 j, & 1 \leq i \leq m-1 \text { and } i \text { is odd } \\
& 1 \leq j \leq n \\
(4 i-5)(2 n-1)+6 n+2 j-6, & 1 \leq i \leq m-1 \text { and } i \text { is even } \\
& 1 \leq j \leq n\end{cases}
\end{aligned}
$$

and

$$
f^{*}\left(w_{i, j} u_{i+1, j}\right)= \begin{cases}(4 i-1)(2 n-1)+6 n-6 j, & 1 \leq i \leq m-1 \text { and } i \text { is odd } \\ (4 i-1)(2 n-1)+6 j-6, & 1 \leq j \leq n \\ & 1 \leq i \leq m-1 \text { and } i \text { is even, } \\ & 1 \leq j \leq n\end{cases}
$$

Hence, $f$ is an odd sum labeling. The odd sum labeling is shown in the following Figure 3.

Proposition 2.8. Let $G$ be a graph obtained by duplicating a vertex in $P_{n}$. Then $S(G)$ is an odd sum graph.

Proof. Let $v_{1}, v_{2}, \ldots, v_{n}$ be the vertices of $P_{n}$.

Case (i). $v_{1}^{\prime}$ or $v_{n}^{\prime}$ is the duplicating vertex of $v_{1}$ (or $v_{n}$ ) in $P_{n}$. Let $u_{i}$, $1 \leq i \leq n-1$ be the subdividing vertices of the edge $v_{i} v_{i+1}$ and $u_{1}^{\prime}$ be the subdividing vertex of the edge $v_{1}^{\prime} v_{2}$. 


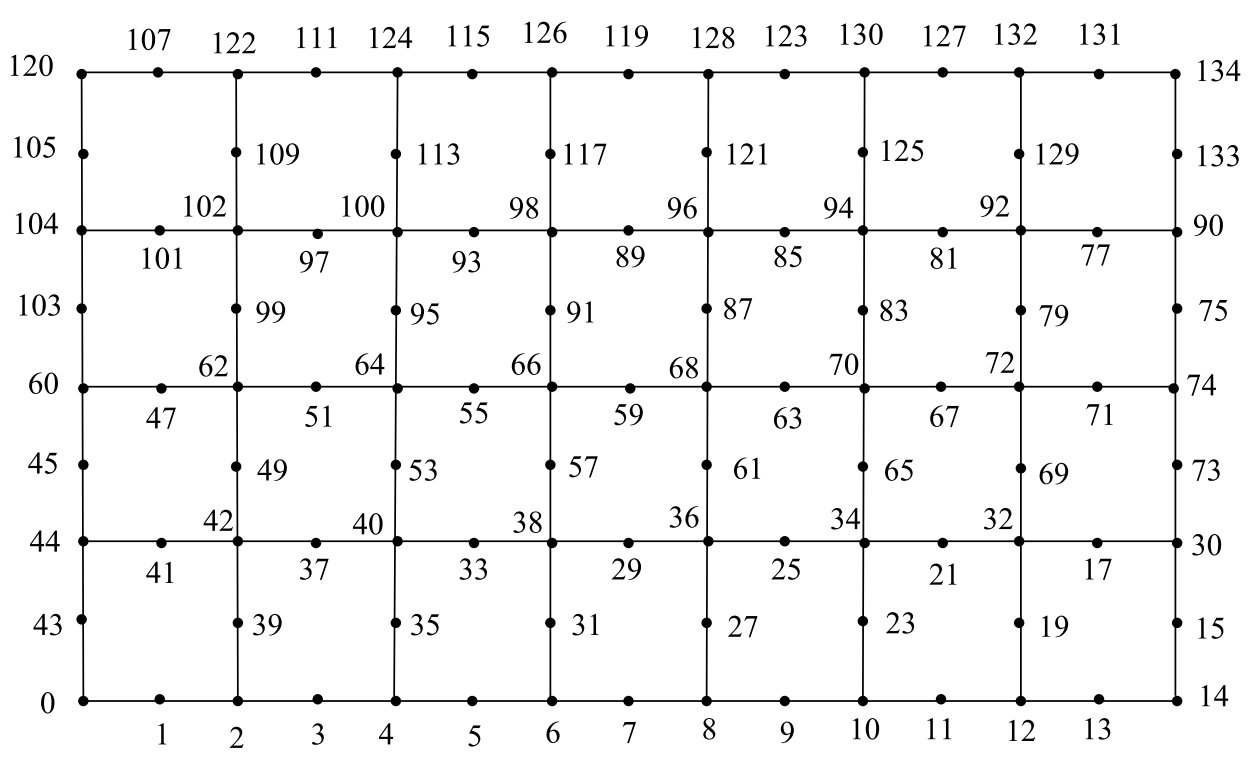

Figure 3. $S\left(P_{5} \times P_{8}\right)$.

Subcase (i). $n$ is odd.

The vertex labeling $f: V(S(G)) \rightarrow\{0,1,2, \ldots, 2 n\}$ is defined as follows:

$$
\begin{aligned}
& f\left(v_{1}^{\prime}\right)=0, f\left(u_{1}^{\prime}\right)=1, f\left(v_{1}\right)=4, f\left(v_{2}\right)=2, f\left(v_{3}\right)=6 \\
& f\left(v_{i}\right)= \begin{cases}2 i-2, & 4 \leq i \leq n \text { and } i \text { is odd } \\
2 i+2, & 4 \leq i \leq n \text { and } i \text { is even }\end{cases} \\
& f\left(u_{1}\right)=3, f\left(u_{2}\right)=7, f\left(u_{3}\right)=5, f\left(u_{4}\right)=9 \text { and } \\
& f\left(u_{i}\right)= \begin{cases}2 i+3, & 5 \leq i \leq n-1 \text { and } i \text { is odd } \\
2 i-1, & 5 \leq i \leq n-1 \text { and } i \text { is even. }\end{cases}
\end{aligned}
$$

The induced edge labeling $f^{*}$ is obtained as follows:

$$
\begin{aligned}
f^{*}\left(v_{1}^{\prime} u_{1}^{\prime}\right) & =1, f^{*}\left(u_{1}^{\prime} v_{2}\right)=3 \\
f^{*}\left(v_{i} u_{i}\right) & =\left\{\begin{array}{ll}
2 i+5, & 1 \leq i \leq 3 \\
4 i+3, & i=4 \\
4 i+1, & 5 \leq i \leq n-1
\end{array}\right. \text { and } \\
f^{*}\left(u_{i} v_{i+1}\right) & = \begin{cases}5, & i=1 \\
2 i+3, & 2 \leq i \leq 4 \\
4 i+7, & 5 \leq i \leq n-1 \text { and } i \text { is odd } \\
4 i-1, & 5 \leq i \leq n-1 \text { and } i \text { is even. }\end{cases}
\end{aligned}
$$

Thus, $f$ is an odd sum labeling. 
Subcase (ii). $n$ is even.

The vertex labeling $f: V(S(G)) \rightarrow\{0,1,2, \ldots, 2 n\}$ is defined as follows:

$$
\begin{aligned}
f\left(v_{1}^{\prime}\right) & =0, f\left(u_{1}^{\prime}\right)=1, f\left(v_{1}\right)=3, f\left(v_{2}\right)=2, \\
f\left(v_{i}\right) & = \begin{cases}2 i+2, & 3 \leq i \leq n \text { and } i \text { is odd } \\
2 i-2, & 3 \leq i \leq n \text { and } i \text { is even }\end{cases} \\
f\left(u_{1}\right) & =4 \text { and } \\
f\left(u_{i}\right) & = \begin{cases}2 i+3, & 2 \leq i \leq n-1 \text { and } i \text { is even } \\
2 i-1, & 2 \leq i \leq n-1 \text { and } i \text { is odd. }\end{cases}
\end{aligned}
$$

The induced edge labeling $f^{*}$ is obtained as follows:

$$
\begin{aligned}
f^{*}\left(v_{1}^{\prime} u_{1}^{\prime}\right) & =1 \\
f^{*}\left(u_{1}^{\prime} v_{2}\right) & =3, \\
f^{*}\left(v_{i} u_{i}\right) & = \begin{cases}7, & i=1 \\
2 i+5, & 2 \leq i \leq n-1\end{cases} \\
\text { and } f^{*}\left(u_{i} v_{i+1}\right) & = \begin{cases}5, & i=1 \\
4 i+7, & 2 \leq i \leq n-1 \text { and } i \text { is even } \\
4 i-1, & 3 \leq i \leq n-1 \text { and } i \text { is odd. }\end{cases}
\end{aligned}
$$

Thus, $f$ is an odd sum labeling.

Case (ii). $\quad v_{i}^{\prime}$ is the duplicating vertex of $v_{i}$ in $P_{n}, 2 \leq i \leq n-1$.

Let $u_{i}, 1 \leq i \leq n-1$ be the subdividing vertex of the edge $v_{i} v_{i+1}$ and $u_{i}^{\prime}$, $u_{i}^{\prime \prime}$ be the subdividing vertices of the edges $v_{i-1} v_{i}^{\prime}$ and $v_{i}^{\prime} v_{i+1}$ respectively. The vertex labeling $f: V(S(G)) \rightarrow\{0,1,2, \ldots, 2 n+2\}$ is defined as follows:

$$
\begin{aligned}
f\left(v_{j}\right) & = \begin{cases}2 j-1, & 1 \leq j \leq i-1 \\
2 j+1, & i \leq j \leq n\end{cases} \\
f\left(u_{j}\right) & = \begin{cases}2 j-2, & 1 \leq j \leq i-2 \\
2 j+4, & i-1 \leq j \leq n-1\end{cases} \\
f\left(v_{i}^{\prime}\right) & =2 i-1, f\left(u_{i}^{\prime}\right)=2 i-4, \text { and } f\left(u_{i}^{\prime \prime}\right)=2 i-2 .
\end{aligned}
$$

The induced edge labeling $f^{*}$ is obtained as follows:

$$
\begin{aligned}
& f^{*}\left(v_{j} u_{j}\right)= \begin{cases}4 j-3, & 1 \leq j \leq i-2 \\
4 i-1, & j=i-1 \\
4 j+5, & 1 \leq j \leq n-1\end{cases} \\
& f^{*}\left(u_{j} v_{j+1}\right)= \begin{cases}4 j-1, & 1 \leq j \leq i-2 \\
4 j+7, & i-1 \leq j \leq n-1\end{cases} \\
& f^{*}\left(v_{i-1} u_{i}^{\prime}\right)=4 i-7, f^{*}\left(u_{i}^{\prime} v_{i}^{\prime}\right)=4 i-5, f^{*}\left(v_{i}^{\prime} u_{i}^{\prime \prime}\right)=4 i-3, \text { and } f^{*}\left(u_{i}^{\prime \prime} v_{i+1}\right)=4 i+1 .
\end{aligned}
$$

Thus, $f$ is an odd sum labeling.

Proposition 2.9. Let $G$ be a graph obtained by duplicating a vertex in $C_{n}$. Then $S(G)$ is an odd sum graph. 
Proof. Let $v_{1}, v_{2}, \ldots, v_{n}$ be the vertices of $C_{n}$ and let $v_{2}^{\prime}$ be the duplicating vertex of $v_{2}$ in $C_{n}$. Let $u_{i}, 1 \leq i \leq n-1$ and let $u_{n}$ be the subdividing vertices of the edges $v_{i} v_{i+1}$ and $v_{n} v_{1}$ respectively and $u_{2}^{\prime}, u_{2}^{\prime \prime}$ be the subdividing vertices of the edges $v_{1} v_{2}^{\prime}$ and $v_{2}^{\prime} v_{3}$ respectively.

Case (i). $\quad n$ is odd and $n \geq 7$.

The vertex labeling $f: V(S(G)) \rightarrow\{0,1,2, \ldots, 2 n+4\}$ is defined as follows:

$$
\begin{aligned}
& f\left(v_{1}\right)=2 \\
& f\left(v_{i}\right)= \begin{cases}2 i+2, & 2 \leq i \leq \frac{n-1}{2} \\
2 i+4, & \frac{n+1}{2} \leq i \leq n\end{cases} \\
& f\left(u_{i}\right)= \begin{cases}2 i+1, & 1 \leq i \leq 2 \\
2 i+3, & 3 \leq i \leq n\end{cases} \\
& f\left(v_{2}^{\prime}\right)=0, f\left(u_{2}^{\prime}\right)=1, \text { and } f\left(u_{2}^{\prime \prime}\right)=7 .
\end{aligned}
$$

The induced edge labeling $f^{*}$ is obtained as follows:

$$
\begin{aligned}
f^{*}\left(v_{i} u_{i}\right) & = \begin{cases}5, & i=1 \\
11, & i=2 \\
4 i+5, & 3 \leq i \leq \frac{n-1}{2} \\
4 i+7, & \frac{n+1}{2} \leq i \leq n\end{cases} \\
f^{*}\left(u_{i} v_{i+1}\right) & = \begin{cases}4 i+5, & 1 \leq i \leq 2 \\
4 i+7, & 3 \leq i \leq \frac{n-3}{2} \\
4 i+9, & \frac{n-1}{2} \leq i \leq n-1\end{cases} \\
f^{*}\left(u_{n} v_{1}\right) & =2 n+5, f^{*}\left(v_{1} u_{2}^{\prime}\right)=3, f^{*}\left(u_{2}^{\prime} v_{2}^{\prime}\right)=1, f^{*}\left(v_{2}^{\prime} u_{2}^{\prime \prime}\right)=7 \\
\text { and } f^{*}\left(u_{2}^{\prime \prime} v_{3}\right) & =15 .
\end{aligned}
$$

Thus, $f$ is an odd sum labeling.

Case (ii). $n$ is even and $n \geq 6$.

The vertex labeling $f: V(\bar{S}(G)) \rightarrow\{0,1,2, \ldots, 2 n+4\}$ is defined as follows:

$$
\begin{aligned}
& f\left(v_{i}\right)= \begin{cases}2 i+1, & 1 \leq i \leq \frac{n}{2} \\
2 i+3, & \frac{n}{2}+1 \leq i \leq n\end{cases} \\
& f\left(u_{i}\right)=2 i+4, \quad 1 \leq i \leq n \\
& f\left(v_{2}^{\prime}\right)=1, f\left(u_{2}^{\prime}\right)=2 \text { and } f\left(u_{2}^{\prime \prime}\right)=0 .
\end{aligned}
$$

The induced edge labeling $f^{*}$ is obtained as follows:

$$
\begin{aligned}
f^{*}\left(v_{i} u_{i}\right) & = \begin{cases}4 i+5, & 1 \leq i \leq \frac{n}{2} \\
4 i+7, & \frac{n}{2}+1 \leq i \leq n\end{cases} \\
f^{*}\left(u_{i} v_{i+1}\right) & = \begin{cases}4 i+7, & 1 \leq i \leq \frac{n}{2}-1 \\
4 i+9, & \frac{n}{2} \leq i \leq n-1\end{cases} \\
f^{*}\left(u_{n} v_{1}\right) & =2 n+7, f^{*}\left(v_{1} u_{2}^{\prime}\right)=5, f^{*}\left(u_{2}^{\prime} v_{2}^{\prime}\right)=3, f^{*}\left(v_{2}^{\prime} u_{2}^{\prime \prime}\right)=1
\end{aligned}
$$

and $f^{*}\left(u_{2}^{\prime \prime} v_{3}\right)=7$. 
Thus, $f$ is an odd sum labeling.

The odd sum labeling for $G$ when $n=3,4,5$ are given as follows in the Figure 4 .
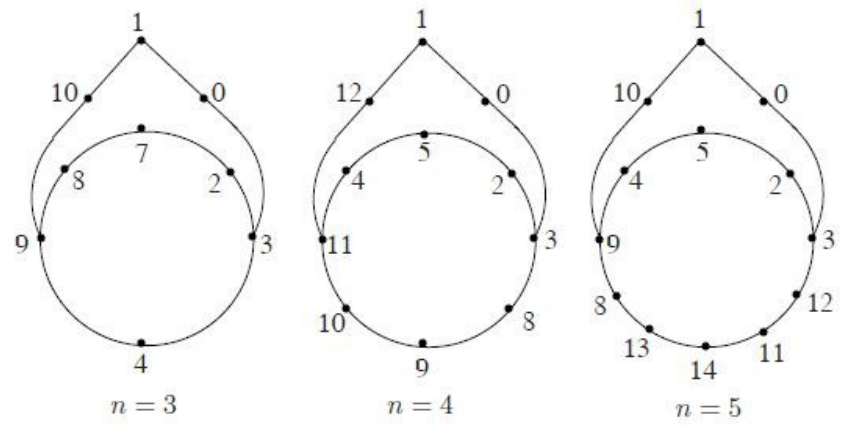

Figure 4

\section{REFERENCES}

[1] F. Buckley and F. Harary, Distance in graphs, Addison-Wesley, Reading, 1990.

[2] R. Balakrishnan, A. Selvam and VY்egnanarayanan, On felicitous labelings of graphs, Graph Theory and its Applications, (1996), 47-61.

[3] J. A. Gallian, A dynamic survey of graph labeling, The Electronic Journal of Combinatorics, 17 (2011), \# DS6.

[4] K. Manickam and M. Marudai, Odd mean labelings of graphs, Bulletin of Pure and Applied Sciences, 25E(1) (2006), 149-153.

[5] R. Ponraj, J. Vijaya Xavier Parthipan and R. Kala, Some results on pair sum labeling of graphs, International Journal of Mathematical Combinatorics, 4 (2010), 53-61.

[6] S. Somasundaram and R. Ponraj, Mean labelings of graphs, National Academy Science Letter, 26 (2003), 210-213.

[7] S. Avadayappan and R. Vasuki, Some results on mean graphs, Ultra Scientist of Physical Sciences, 21(1)M (2009), 273-284.

${ }^{1}$ Department of Mathematics,

Mepco Schlenk Engineering College,

SivAKASI-626 005, TAMILNADU, INDIA.

E-mail address: psarockiaraj@gmail.com

${ }^{2}$ Department of Mathematics,

Kamaraj College of Engineering and Technology,

VirudhunagaR- 626 001, TAmilnadu, INDIA.

E-mail address: mahajai1979@gmail.com

${ }^{3}$ Department of Mathematics,

M.D.T. Hindu College,

Tirunelveli-627 010, TAmilnadu, INDIA.

E-mail address: vasuhe2010@gmail.com 